\title{
Searching for a better definition of robotic surgery: is it really different from laparoscopy?
}

\author{
Andrew A. Gumbs, Belinda De Simone, Elie Chouillard \\ Departement de Chirurgie Digestive, Centre Hospitalier Intercommunal de Poissy/Saint-Germain-en-Laye, Poissy 78300, \\ France.
}

Correspondence to: Prof. Andrew A. Gumbs, Departement de Chirurgie Viscérale et Digestive, Centre Hospitalier de POISSYSAINT GERMAIN, 10 rue du Champ Gaillard, Poissy 78300, France. E-mail: aagumbs@gmail.com

How to cite this article: Gumbs AA, De Simone B, Chouillard E. Searching for a better definition of robotic surgery: is it really different from laparoscopy? Mini-invasive Surg 2020;4:90. http://dx.doi.org/10.20517/2574-1225.2020.110

Received: 26 Nov 2020 Accepted: 30 Nov 2020 Published: 10 Dec 2020

Academic Editor: Giulio Belli Copy Editor: Cai-Hong Wang Production Editor: Jing Yu

Although both laparoscopic surgery and robotic surgery are minimally invasive techniques, the hope for robotic surgery is that it represents an evolution of minimally invasive technology that will improve the precision of surgeons movements in ever increasingly narrow and small anatomic spaces. It is widely believed that robotic technology works as a filter for the involuntary tremors of the surgeon, theoretically resulting in a minimization of involuntary inaccuracies, thus helping surgeons to further perfect their art. That robotic surgery is a natural evolution of minimally invasive surgery is not questioned; however, the veritable explosion of robotic enhancement begs the questions: Are all surgical robots created equal? What should be considered robotic surgery and what should be considered robot-assisted?

The meaning of the words robot and robotics are surprisingly complex. The etymology of robot comes from the Slavic word "robot" that means servitude, servant, and disturbingly slave. It first appeared in print in 1920 in the play R.U.R. (Rossum's Universal Robots) about a factory that makes androids and was written by the Czech writer Karel Čapek ${ }^{[1]}$. Isaac Asimov is then credited with coining the term robotics in a short story tilted "Liar!" that was first published in $1941^{[2]}$. Since then, the term robot has taken on a number of meanings with its main definition being a machine or device that does the work of a human either autonomously or under computer control.

Robotics has become a field of engineering that utilizes computer science to design, manufacture, operate, and utilize robots. It has become an interdisciplinary field that uses aspects of electronic, computer, mechanical, and information engineering. The field of robotics has innumerable potential applications,

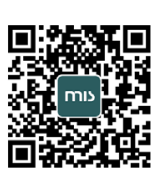



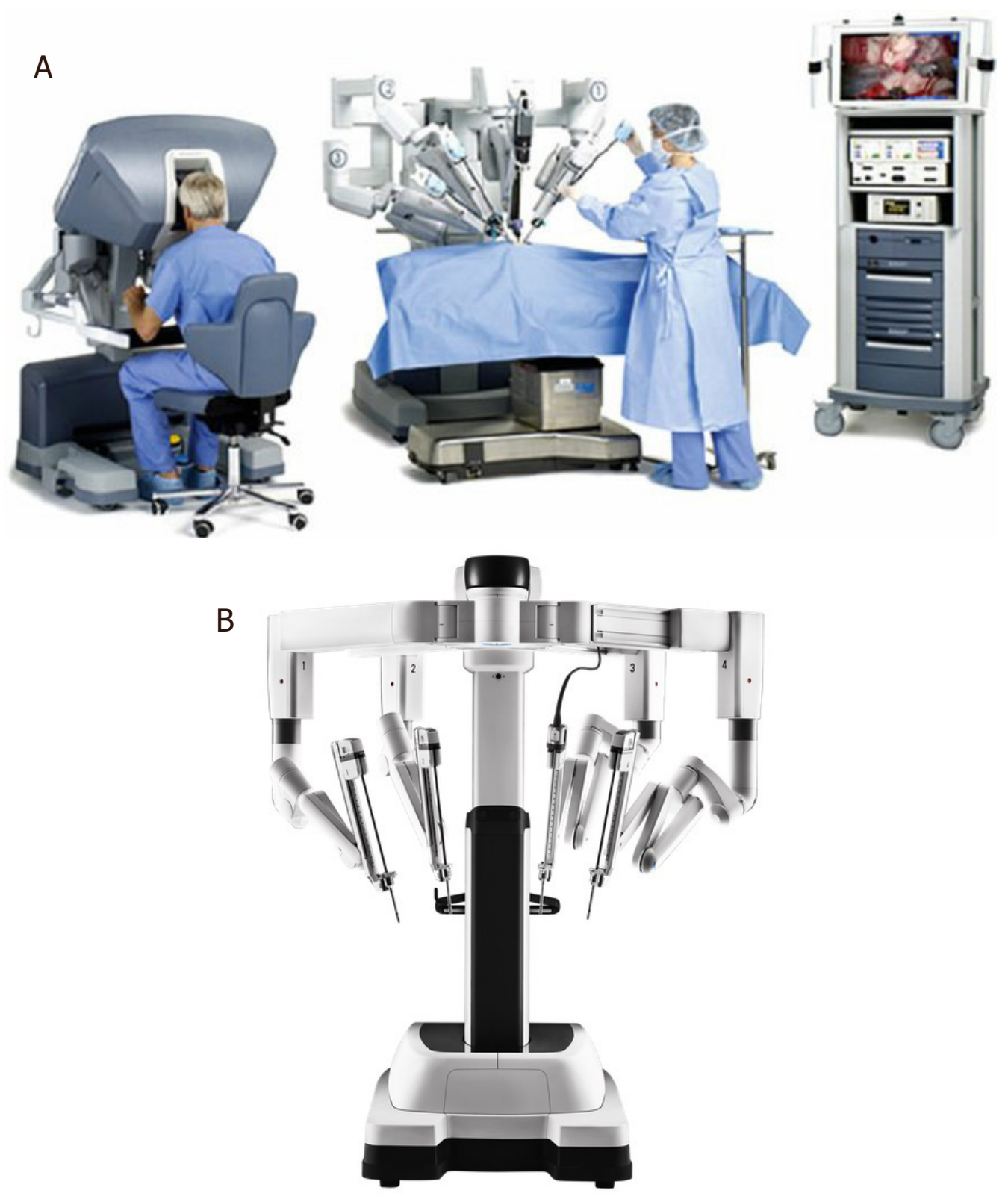

Figure 1. A: da Vinci complete surgical robotic systems have three major components: (1) the surgeon's console; (2) a surgical cart with the robotic arms and end-effectors; and (3) the visual cart (copyright Intuitive Surgical International - reproduced courtesy of the manufacturer); B: the latest iteration of the surgical cart for the da Vinci Xi Surgical System (da Vinci, Intuitive Surgical, Sunnyvale, CA, USA)

but it has been divided into five broad fields: sensors, programming, mobility, human-robot interface, and manipulation. It has also been divided into four broad divisions: bio, industrial, mobile, and aerial. Currently, robotic surgery would seem to fall in the bio division and mainly in the field of manipulation. Perhaps the clearest definition of robots is the one published by the American Institute of Robotics in 1979, "a robot is a reprogrammable, multifunctional manipulator designed to move material, parts, tools, or specialized devices through variable programmed motions for the performance of a variety of tasks" ${ }^{\text {"[1] }}$.

Although the da Vinci robot (da Vinci, Intuitive Surgical, Sunnyvale, CA, USA) has come to dominate the field of robotic surgery [Figure 1], the field of robot-assisted surgery was initially popularized with the robotically-controlled laparoscope holder AESOP (Computer Motion, Inc., Sunnyvale, CA, USA) in 


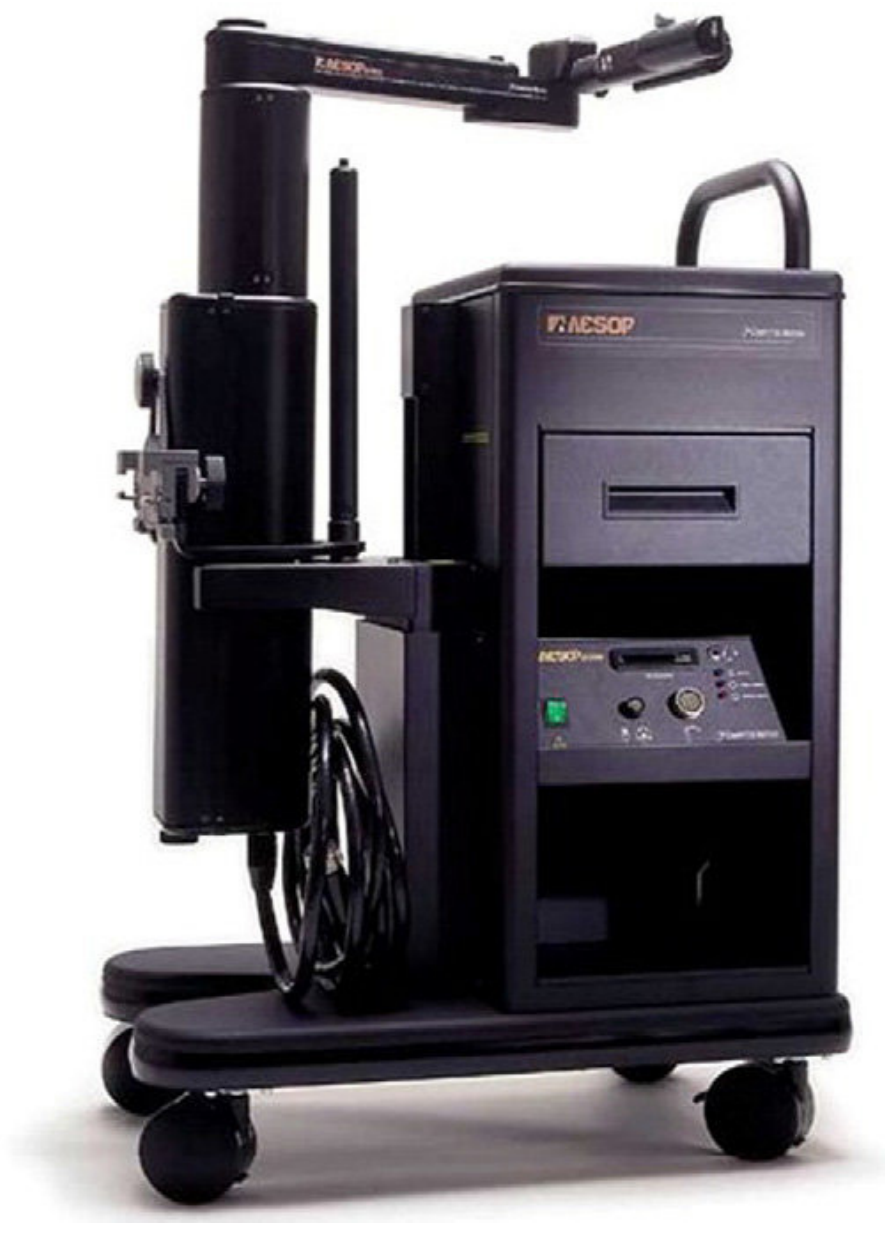

Figure 2. The AESOP robotic laparoscope holder (Computer Motion, Inc., Sunnyvale, CA, USA)

the mid-1990s ${ }^{[3]}$ [Figure 2]. This device was so well-liked that it became a victim of its efficacy, and the company was purchased by Intuitive Surgical and promptly shelved, thus eliminating any competition. Nonetheless, this remote-controlled robot is widely considered the first robot used in minimally invasive abdominal surgery, yet surgeries done with it are not even considered robotically-assisted procedures by most surgeons.

Another robotically-controlled laparoscope holder called ViKY (short for Video-endosKopY; ViKY, Endocontrol, Grenoble, France) then came on the market ${ }^{[4]}$ [Figure 3]. Unlike AESOP or the da Vinci, this robotically-controlled laparoscope holder is autoclavable and can be sterilized. Endocontrol then developed hand-held 'robotic' instruments that have additional degrees of articulation that are really just motorized laparoscopic instruments (JaiMY, Endocontrol, Grenoble, France) ${ }^{[5]}$ [Figure 4]. These two devices were developed so that surgeons could overcome the loss of haptics that exists with the da Vinci Robot, specifically the loss of the sensation of touch. Other hand-held instruments with end-effectors and increased degrees of freedom exist; however, unlike JaiMY, these devices are fully powered by the force of the surgeon and have no powered motors.

Robotic surgery is traditionally defined as any surgery done with a complete robotic surgical system. Up until recently, the only complete system was the da Vinci Surgical System [Figure 1]. It was originally developed for the military so that surgeons could remotely do open surgery on wounded soldiers in the field; the device was retrofitted for minimally invasive surgery as this was more marketable. During these 


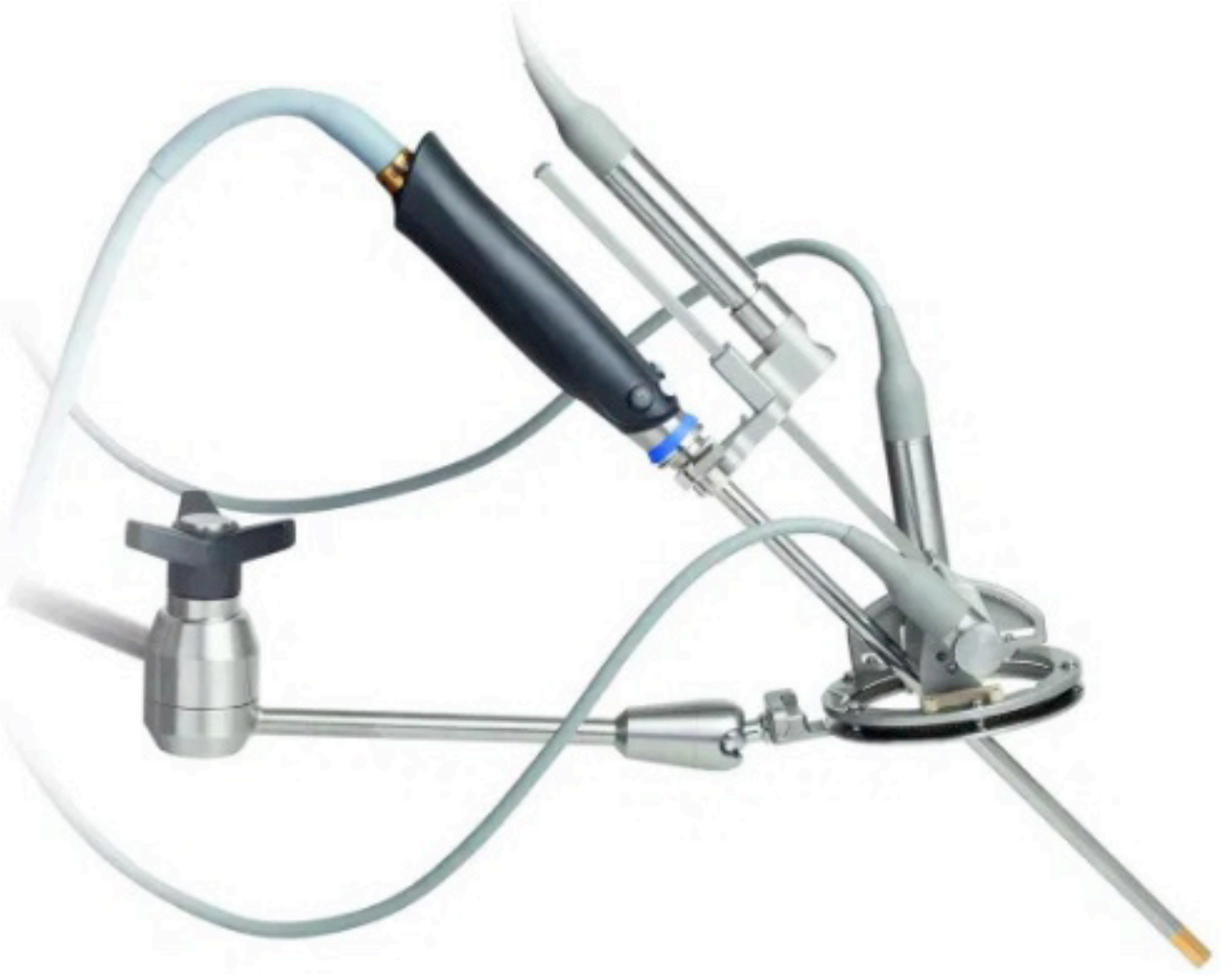

Figure 3. VideoendosKopY (ViKY) robotically-controlled laparoscope holder (ViKY, Endocontrol, Grenoble, France)

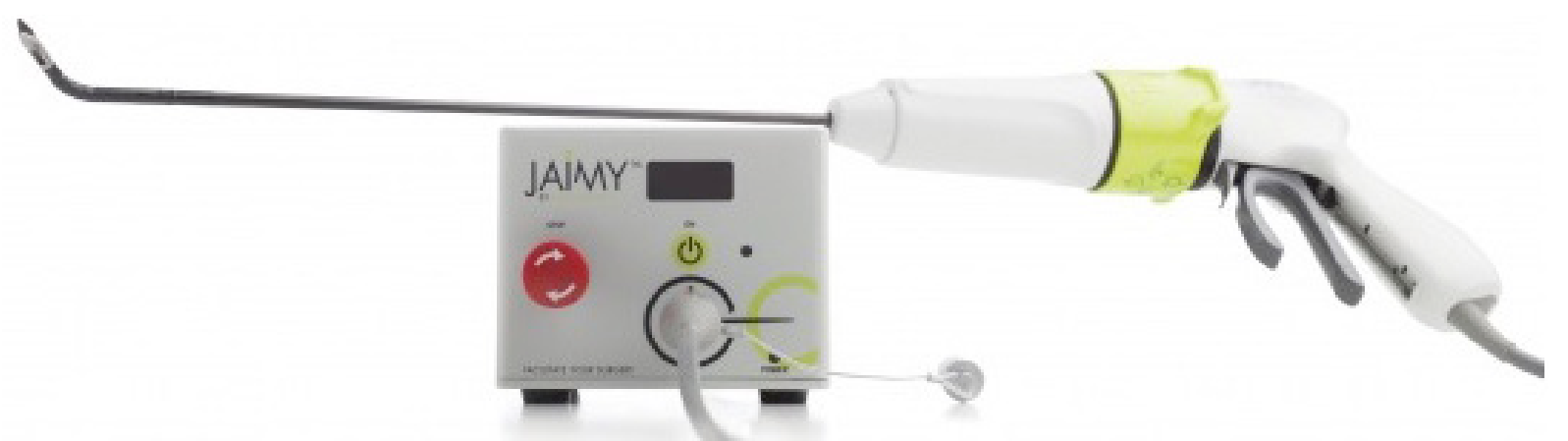

Figure 4. Articulating 5-mm laparoscopic instrument with motorized control (JAIMY-EN, Endocontrol, Grenoble, France)

procedures, a surgeon sits at a console several feet away from the patient, and the motorized effector arms of the robot are the ones in actual contact with the patient ${ }^{[1]}$. The operating surgeon is not wearing a sterile gown or gloves and only the robotic arms and surgical assistant are in contact with the patient. A telemanipulator is a remotely-controlled device that enables the surgeon to control surgical instruments using manipulators and motorized end-effectors. During the Lindbergh Operation in 2001, when the first Trans-Atlantic minimally invasive surgery was done, a telemanipulator was also used, but an additional computerized system was necessary to control the end effectors and robotic arms across such a great distance $^{[6]}$.

The bright future of complete surgical systems is perhaps best highlighted by the development of competitors to the da Vinci robot. The Versius robot (Versius Robotics, CMR, Cambridge, UK) has a computer interface to enable haptic feedback [Figure 5]. A notable weakness of the earlier da Vinci robots 


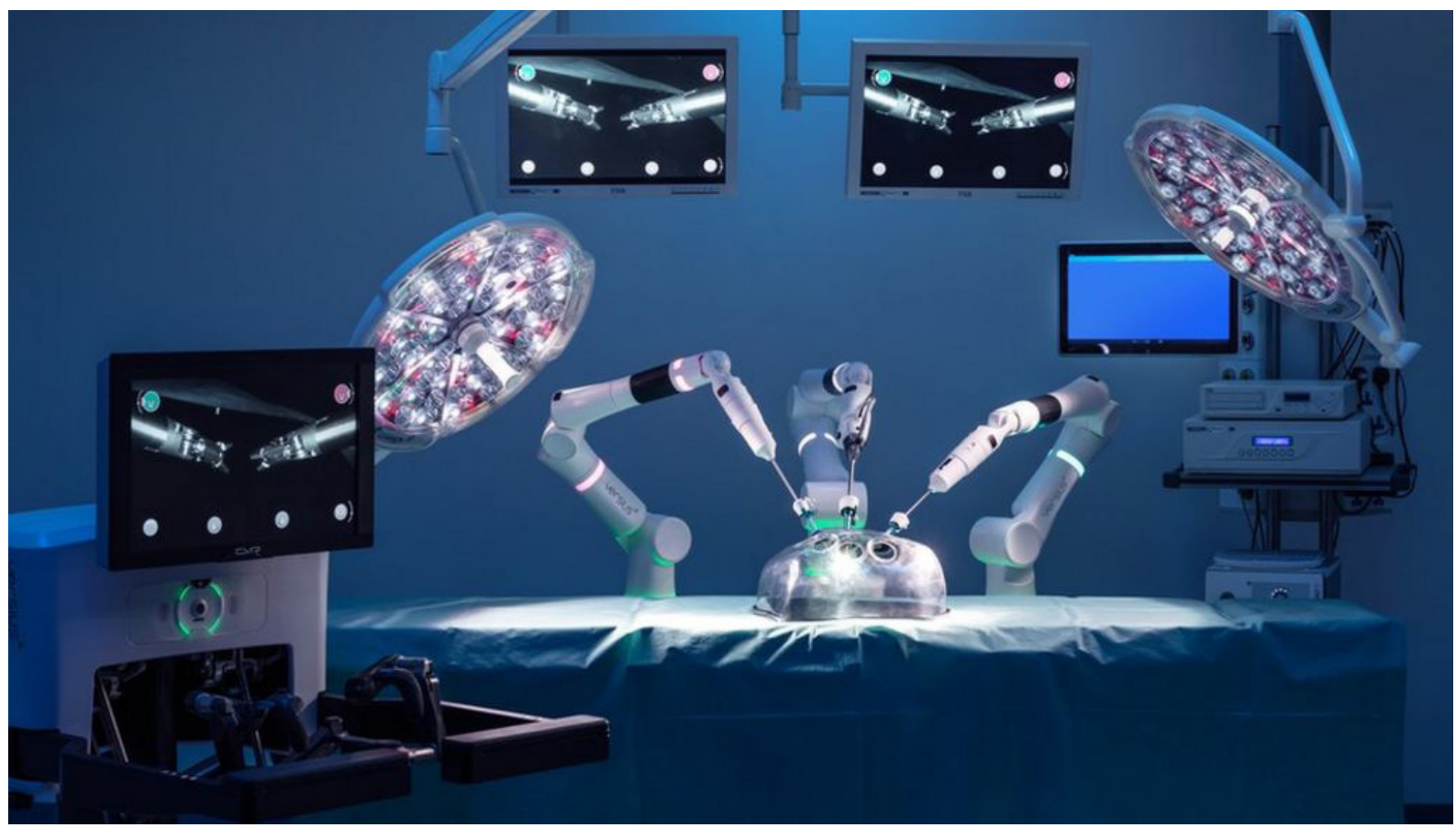

Figure 5. The Versius complete surgical system with surgical console, robotic arms, and laparoscopic tower (Versius Robotics, CMR, Cambridge, UK)

and one of the main reasons certain minimally invasive surgeons have not embraced this technology is that they are waiting for a robot with haptics ${ }^{[7]}$. Notably, the initial generation Versius will also not have haptics ${ }^{[8,9]}$. Medtronic, one of the largest surgical instrument companies, has even developed a complete surgical system called Hugo (Hugo Robot, Medtronic Inc., Dublin, Ireland).

With the general surgeon's current definition of robotic surgery, the robot is not autonomous and does not perform any actions automatically. Instead, the instruments move either through the action of a telemanipulator with motorized end-effectors or through computer control. In short, robotic surgery seems to fall well short of the definition proposed by the American Institute of Robotics ${ }^{[1]}$. On the contrary, in spinal surgery (Mazor Robotics, Mazor Robotics, Inc., Caesarea, Israel), radiation therapy with the Cyberknife (Cyberknife System, Sunnyvale, CA, USA), and head and neck surgery (Flex Robotics System, MedRobotics, Raynham, MA), there are several robots that also have some degree of automation ${ }^{[2,10-12]}$. Ultimately, it must be remembered that the logical conclusion of developing robotic surgery will probably result in either partial or total automation of operations even in general surgery.

Another reason that the term robotic surgery is difficult to define is that several devices used in abdominal surgery have automatic motorized components. There is a hand-held stapler called the iDrive (iDrive, Medtronic Inc., Dublin, Ireland) with automatic motorized stapling that if used could technically define a procedure as being robotically-assisted [Figure 6]. Theoretically, an open colectomy where a surgeon uses the iDrive could be considered a robotically-assisted procedure. Furthermore, although some so-called robotic cases use a complete "robotic" surgical system for the majority of the procedure, some procedures use a hybrid approach. For instance, should a minimally invasive esophagectomy that had its abdominal portion done laparoscopically, but the thoracic portion done with the da Vinci robot, be considered robotic, robot-assisted, or is minimally invasive a better term? Is there a percentage of a case that needs to be done with the robot before it should be considered laparoscopic, robotic, or robot-assisted? What about robotically-assisted Whipple procedures where the pancreatic head resection is done laparoscopically, 


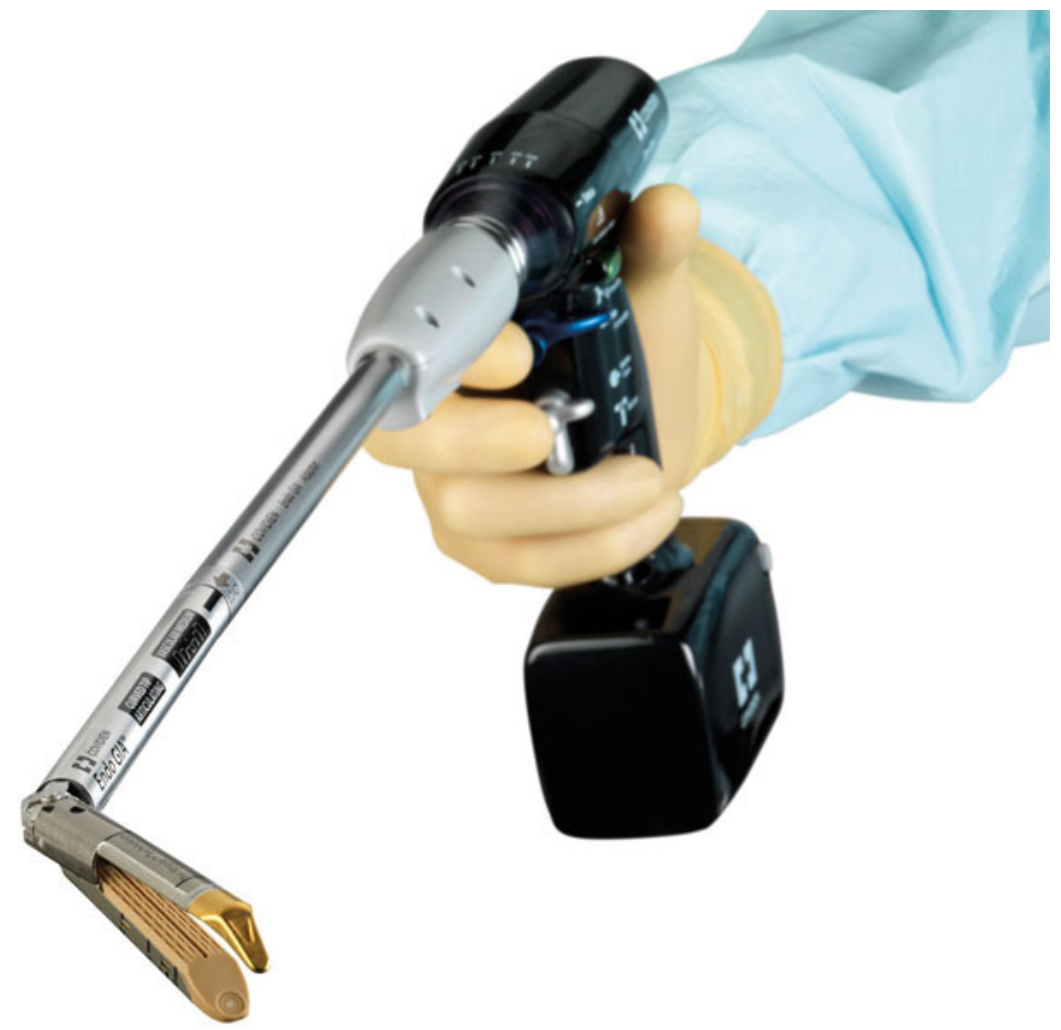

Figure 6. Automatic motorized gastrointestinal stapler device (iDrive, Medtronic Inc., Dublin, Ireland)

but the reconstruction is done robotically or vice versa ${ }^{[13]}$ ? What if the reconstruction is done though a mini-laparotomy? If a robotically-controlled laparoscope holder is used for a totally laparoscopic Whipple procedure should it be considered robotically-assisted ${ }^{[14,15]}$ ?

Notably, new techniques of hernia repair that obviate the need for entering into the abdomen at all such as the Trentino Hernia Team (THT) technique may make the robot superfluous for many midline hernia repairs ${ }^{[16]}$. Currently, open surgery is still the fundamental foundation of abdominal surgery regardless of the approach used, particularly for the management of catastrophic injuries and complications. As a result, in this Special Issue, some authors will discuss the management of certain sequelae and/or complications of robotic and laparoscopic minimally invasive surgery that cannot currently be managed minimally invasively. This highlights the possibility that, although some general surgical procedures, such as ileostomy takedown, can never be done minimally invasively, they could theoretically be done with the aid of a robot. The original impetus for the da Vinci cannot be ignored: engineers wanted to create a robot that could do even open surgery, and surgeons must be prepared for the possibility that one day this may become a reality. This possibility is made more clear when we consider that robots designed to function as scrub nurses have already been developed ${ }^{[17]}$.

Currently, the complete surgical "robotic" systems seem to be more of a "motion-control" system and not a fully robotic or "reprogrammable" surgical system ${ }^{[2]}$. Nonetheless, these complete systems will continue to be beneficial for surgeons, particularly for pathology in small spaces such as the pelvis, which has been elucidated by the explosion of robotic radical prostatectomy. With the continued evolution of robotic platforms such as the da Vinci Single-Site Platform (da Vinci Single Site Technology, Intuitive Surgical, Sunnyvale, CA, USA) [Figure 7], it is impossible to deny the future potential of robotic surgery. This collection of invited manuscripts from international leaders in the field of robotic and laparoscopic surgery 

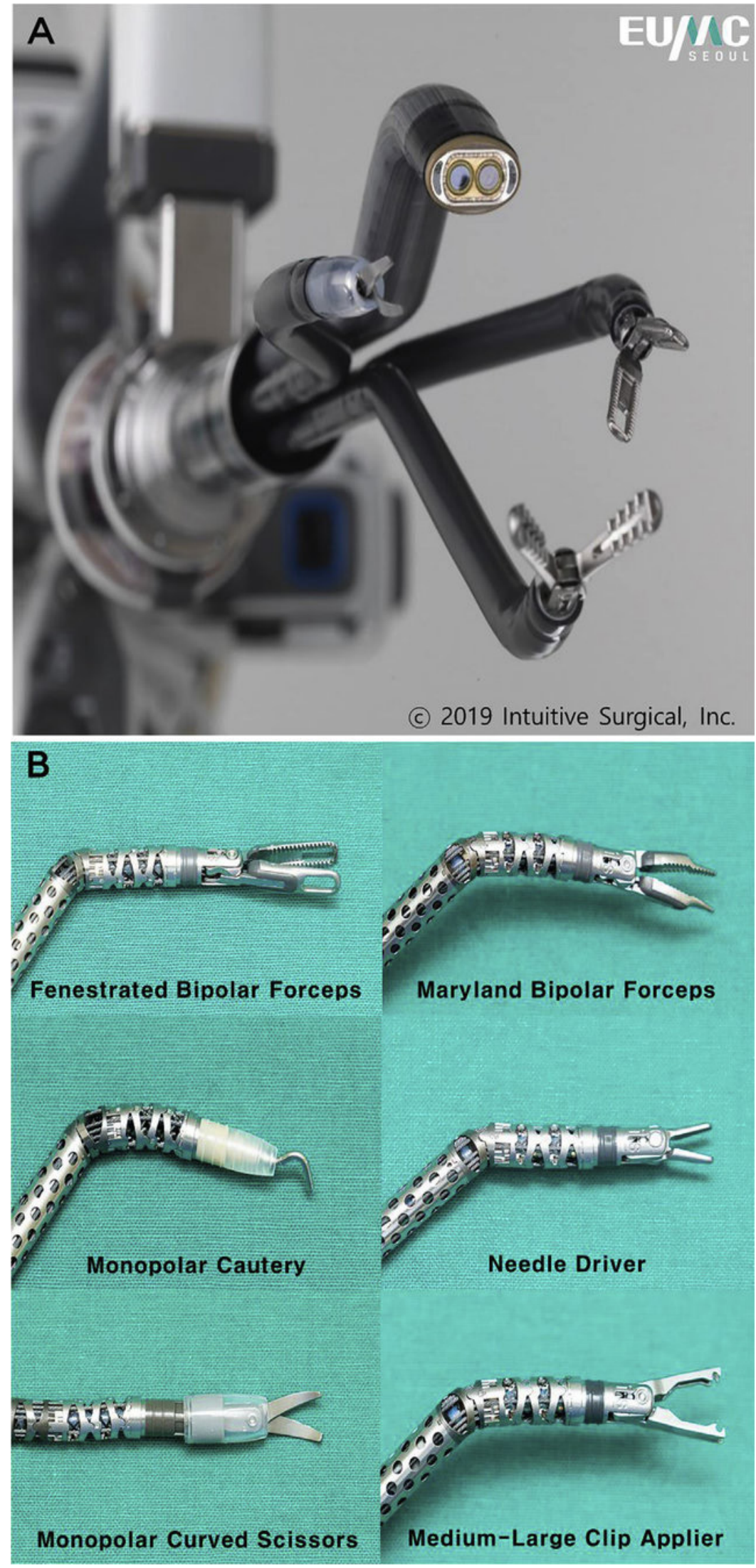

Figure 7. A: da Vinci Single Port (SP) arm (da Vinci, Intuitive Surgical, Sunnyvale, CA, USA); B: available instruments for the da Vinci SP (da Vinci, Intuitive Surgical, Sunnyvale, CA, USA) 
will hopefully shed some light on the question as to what the relevant definition of robotic or robot-assisted surgery should be. It is increasingly clear that surgeons will need to be fluent in open, laparoscopic (including endoscopic and thoracoscopic), and "robotic" techniques, and that all three of these modalities are simply what it means to be a modern surgeon.

\section{DECLARATIONS}

\section{Authors' contributions}

Drafting of manuscript and editing: Gumbs AA

Editing and provided administrative support: De Simone B, Chouillard E

\section{Availability of data and materials}

Not applicable.

\section{Financial support and sponsorship}

None.

\section{Conflicts of interest}

All authors declared that there are no conflicts of interest.

\section{Ethical approval and consent to participate}

Not applicable.

\section{Consent for publication}

Not applicable.

\section{Copyright}

(c) The Author(s) 2020.

\section{REFERENCES}

1. Moran ME. Rossum's universal robots: not the machines. J Endourol 2007;21:1399-402.

2. Theodore N, Arnold PM, Mehta AI. Introduction: the rise of the robots in spinal surgery. Neurosurg Focus 2018;45:Intro.

3. Unger SW, Unger HM, Bass RT. AESOP robotic arm. Surg Endosc 1994;8:1131.

4. Gumbs AA, Crovari F, Vidal C, Henri P, Gayet B. Modified robotic lightweight endoscope (ViKY) validation in vivo in a porcine model. Surg Innov 2007;14:261-4.

5. Bensignor T, Morel G, Reversat D, Fuks D, Gayet B. Evaluation of the effect of a laparoscopic robotized needle holder on ergonomics and skills. Surg Endosc 2016;30:446-54.

6. Marescaux J, Leroy J, Gagner M, et al. Transatlantic robot-assisted telesurgery. Nature 2001;413:379-80.

7. Vibert E, Denet C, Gayet B. Major digestive surgery using a remote-controlled robot: the next revolution. Arch Surg 2003;138:1002-6.

8. Kelkar D, Borse MA, Godbole GP, Kurlekar U, Slack M. Interim safety analysis of the first-in-human clinical trial of the Versius surgical system, a new robot-assisted device for use in minimal access surgery. Surg Endosc 2020; doi: 10.1007/s00464-020-08014-4.

9. Puntambekar SP, Goel A, Chandak S, et al. Feasibility of robotic radical hysterectomy (RRH) with a new robotic system. Experience at galaxy care laparoscopy institute. J Robot Surg 2020; doi: 10.1007/s11701-020-01127-x.

10. Adler JR Jr, Chang SD, Murphy MJ, Doty J, Geis P, Hancock SL. The cyberknife: a frameless robotic system for radiosurgery. Stereotact Funct Neurosurg 1997;69:124-8.

11. Lieberman IH, Togawa D, Kayanja MM, et al. Bone-mounted miniature robotic guidance for pedicle screw and translaminar facet screw placement: Part I--Technical development and a test case result. Neurosurgery 2006;59:641-50.

12. Mandapathil M, Greene B, Wilhelm T. Transoral surgery using a novel single-port flexible endoscope system. Eur Arch Otorhinolaryngol 2015;272:2451-6.

13. Gumbs AA, Croner R, Chouillard E. Is robotic pancreatic surgery finally ready for prime-time? Hepatobiliary Surg Nutr 2020;9:650-3.

14. Gumbs AA, Croner R, Rodriguez A, Zuker N, Perrakis A, Gayet B. 200 consecutive laparoscopic pancreatic resections performed with a robotically controlled laparoscope holder. Surg Endosc 2013;27:3781-91.

15. Gumbs AA, Chouillard E, Abu Hilal M, Croner R, Gayet B, Gagner M. The experience of the minimally invasive (MI) fellowship-trained (FT) hepatic-pancreatic and biliary (HPB) surgeon: could the outcome of MI pancreatoduodenectomy for peri-ampullary tumors be better 
than open? Surg Endosc 2020; doi:10.1007/s00464-020-08118-x.

16. Carrara A, Lauro E, Fabris L, Frisini M, Rizzo S. Endo-laparoscopic reconstruction of the abdominal wall midline with linear stapler, the THT technique. Early results of the first case series. Ann Med Surg (Lond) 2018;38:1-7.

17. Jacob M, Li YT, Akingba G, Wachs JP. Gestonurse: a robotic surgical nurse for handling surgical instruments in the operating room. $J$ Robot Surg 2012;6:53-63. 